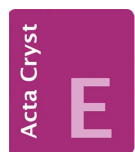

CRYSTALLOGRAPHIC COMMUNICATIONS

ISSN 2056-9890

Received 22 June 2019

Accepted 22 July 2019

Edited by H. Ishida, Okayama University, Japan

${ }^{1}$ This paper is dedicated to Her Royal Highness Princess Chulabhorn Krom Phra Srisavangavadhana of Thailand for her contributions to science on the occasion of her 62th birthday, which fell on July 4th, 2019.

\# Thomson Reuters ResearcherID: A-50852009.

Keywords: crystal structure; hydrazide; molecular conformation; antioxidant; $\alpha$-glucosidase inhibitory.

CCDC reference: 1942396

Supporting information: this article has supporting information at journals.iucr.org/e

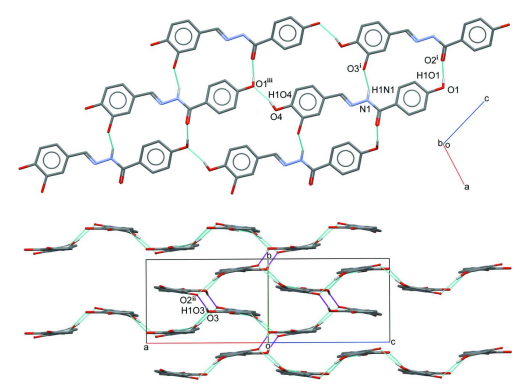

OPEN $\odot$ ACCESS

\section{Crystal structure of $(E)-N^{\prime}$-(3,4-dihydroxybenzyl- idene)-4-hydroxybenzohydrazide ${ }^{1}$}

\author{
Suchada Chantrapromma, ${ }^{a *} \ddagger$ Huey Chong Kwong, ${ }^{b}$ Patcharawadee Prachumrat, \\ Thawanrat Kobkeatthawin, ${ }^{a}$ Tze Shyang Chia ${ }^{\mathrm{c}}$ and Ching Kheng Quah ${ }^{\mathrm{c}}$
}

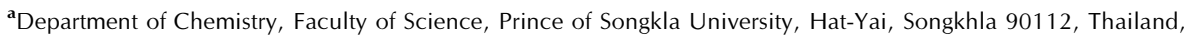
${ }^{\mathbf{b}}$ Department of Chemistry, Faculty of Science, Universiti Putra Malaysia, 43400 UPM Serdang, Selangor Darul Ehsan, Malaysia, and ${ }^{C} X$-ray Crystallography Unit, School of Physics, Universiti Sains Malaysia, 11800 USM, Penang, Malaysia. *Correspondence e-mail: suchada.c@psu.ac.th, suchada_3p@hotmail.com

In the title benzohydrazide derivative, $\mathrm{C}_{14} \mathrm{H}_{12} \mathrm{~N}_{2} \mathrm{O}_{4}$, the azomethine $\mathrm{C}=\mathrm{N}$ double bond has an $E$ configuration. The hydrazide connecting bridge, $(\mathrm{C}=\mathrm{O})-(\mathrm{NH})-\mathrm{N}=(\mathrm{CH})$, is nearly planar with $\mathrm{C}-\mathrm{C}-\mathrm{N}-\mathrm{N}$ and $\mathrm{C}-\mathrm{N}-$ $\mathrm{N}=\mathrm{C}$ torsion angles of $-177.33(10)$ and $-174.98(12)^{\circ}$, respectively. The 4-hydroxyphenyl and 3,4-dihydroxyphenyl rings are slightly twisted, making a dihedral angle of $9.18(6)^{\circ}$. In the crystal, molecules are connected by $\mathrm{N}-\mathrm{H} \cdots \mathrm{O}$ and $\mathrm{O}-\mathrm{H} \cdots \mathrm{O}$ hydrogen bonds into a three-dimensional network, while further consolidated via $\pi-\pi$ interactions [centroid-centroid distances $=3.6480(8)$ and 3.7607 (8) $\AA$ ] . The conformation is compared to those of related benzylidene-4hydroxybenzohydrazide derivatives.

\section{Chemical context}

Hydrazides and hydrazones are important synthons for several transformations and have gained importance because of their various biological and clinical applications (Narasimhan et al., 2010). Benzohydrazide derivatives containing an azomethine $(-\mathrm{NHN}=\mathrm{CH}-)$ group have been reported to possess diverse biological activities such as antitumor (Xia et al., 2007; Kumari \& Bansal, 2018), antioxidant (Aziz et al., 2014), antitubercular and antimicrobial (Maheswari \& Manjula, 2015) and $\alpha$-glucosidase inhibition (Taha et al., 2015) activities. The interesting biological activities of benzohydrazides led us to synthesize several benzohydrazides to study their bioactivities (Fun et al., 2011; Horkaew et al., 2011; Chantrapromma et al., 2016), including the title compound (I), which was found to exhibit antioxidant activity with an $\mathrm{IC}_{50}$ value of $0.035 \pm 0.004 \mathrm{~m} M$ (ascorbic acid used as the reference standard; Thaipong et al., 2006) and $\alpha$-glucosidase inhibitory activity with an $\mathrm{IC}_{50}$ value of $0.014 \pm 0.001 \mathrm{~m} M$ (acarbose as the reference standard; Bachhawat et al., 2011).<smiles>O=C(N/N=C/c1ccc(O)c(O)c1)c1ccc(O)cc1</smiles> 


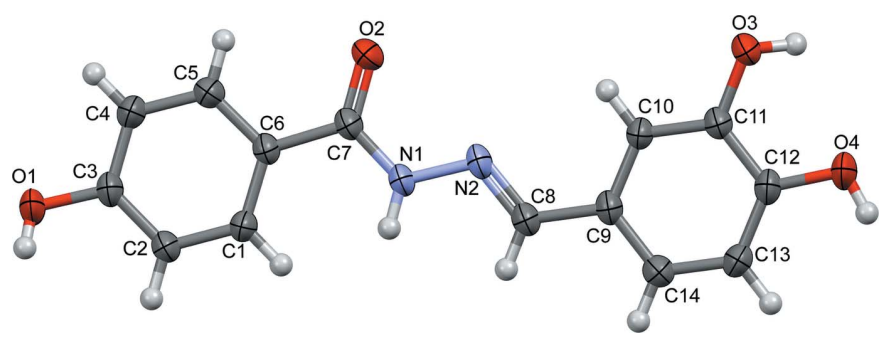

Figure 1

The molecular structure of the title compound with the atom-labelling scheme and displacement ellipsoids at the $50 \%$ probability level.

\section{Structural commentary}

The title hydrazide derivative, (I), consists of a 4-hydroxyphenyl ring, a 3,4-dihydroxyphenyl ring and a hydrazide $(\mathrm{C}=\mathrm{O})-(\mathrm{NH})-\mathrm{N}=(\mathrm{CH})$ connecting bridge (Fig. 1). The $\mathrm{C} 6-\mathrm{C} 7, \mathrm{C} 7-\mathrm{N} 1$ and $\mathrm{C} 8-\mathrm{C} 9$ bond lengths of $1.4861(15)$, 1.3385 (17) and $1.4584(16) \AA$, respectively, confirm their single-bond character, whereas the $\mathrm{C} 7=\mathrm{O} 2$ and $\mathrm{N} 2=\mathrm{C} 8$ bond lengths of 1.2403 (15) and 1.2738 (17) $\AA$, respectively, confirm the presence of the double bonds. The $s p^{2}$-hybridized character of atoms $\mathrm{C} 7$ and $\mathrm{C} 8$ is further supported by the bond angles $\quad \mathrm{C} 6-\mathrm{C} 7-\mathrm{N} 1 \quad\left[116.49(11)^{\circ}\right]$ and $\mathrm{N} 2-\mathrm{C} 8-\mathrm{C} 9$ $\left[120.86(12)^{\circ}\right]$. The bond lengths and angles of the central hydrazide connecting bridge are consistent with those in related structures (Fun et al., 2011; Chantrapromma et al., 2016). The molecule exhibits an $E$ configuration with respect to the azomethine $\mathrm{C}=\mathrm{N}$ double bond. As the torsion angle $\mathrm{C} 6-\mathrm{C} 7-\mathrm{N} 1-\mathrm{N} 2\left[-177.33(10)^{\circ}\right]$ and $\mathrm{C} 7-\mathrm{N} 1-\mathrm{N} 2-\mathrm{C} 8$ $\left[-174.98(12)^{\circ}\right]$ are both in an anti-periplanar conformation, the overall conformation for the hydrazide connecting bridge is almost planar. Furthermore, the 4-hydroxyphenyl and
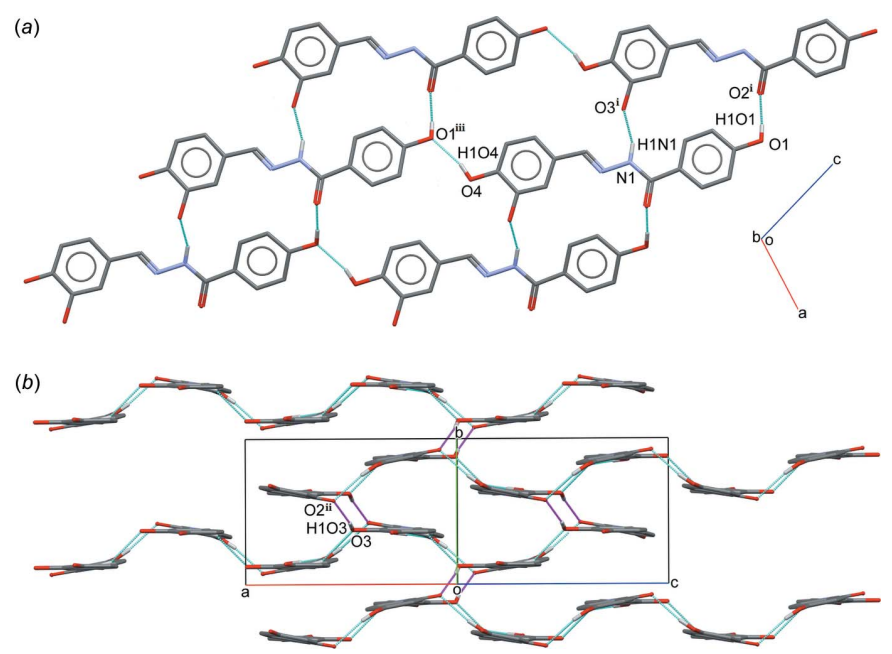

Figure 2

(a) A partial packing diagram of the title compound, showing a twodimensional plate formed by $\mathrm{O}-\mathrm{H} \cdots \mathrm{O}$ and $\mathrm{N}-\mathrm{H} \cdots \mathrm{O}$ hydrogen bonds (cyan dotted lines). [Symmetry codes: (i) $x,-y+\frac{1}{2}, z+\frac{1}{2}$; (iii) $x-1, y$, $z$ - 1.] (b) A partial packing diagram of the title compound with additional $\mathrm{O}-\mathrm{H} \cdots \mathrm{O}$ hydrogen bonds (magenta dotted lines). [Symmetry code: (ii) $-x+1,-y+1,-z$.] Hydrogen atoms not involved in with these interactions are omitted for clarity.
Table 1

Hydrogen-bond geometry $\left(\AA,^{\circ}\right)$.

\begin{tabular}{lllll}
\hline$D-\mathrm{H} \cdots A$ & $D-\mathrm{H}$ & $\mathrm{H} \cdots A$ & $D \cdots A$ & $D-\mathrm{H} \cdots A$ \\
\hline $\mathrm{O} 1-\mathrm{H} 1 O 1 \cdots \mathrm{O} 2^{\mathrm{i}}$ & $0.80(2)$ & $1.92(2)$ & $2.7203(15)$ & $171(2)$ \\
$\mathrm{O} 3-\mathrm{H} 1 O 3 \cdots \mathrm{O} 2^{\mathrm{ii}}$ & $0.88(2)$ & $2.17(2)$ & $3.0276(13)$ & $163(2)$ \\
$\mathrm{O}^{\mathrm{iii}}-\mathrm{H} 1 O 4 \cdots \mathrm{O}^{\mathrm{iii}}$ & $0.82(2)$ & $1.93(2)$ & $2.7379(16)$ & $166(2)$ \\
$\mathrm{N} 1-\mathrm{H} 1 N 1 \cdots 3^{\mathrm{i}}$ & $0.87(2)$ & $2.24(2)$ & $3.0017(16)$ & $146.1(19)$ \\
\hline
\end{tabular}

Symmetry codes: (i) $x,-y+\frac{1}{2}, z+\frac{1}{2}$; (ii) $-x+1,-y+1,-z$; (iii) $x-1, y, z-1$.

3,4-dihydroxyphenyl rings are also coplanar to the corresponding azomethine and carbonyl double bonds, with torsion angles $\mathrm{N} 2-\mathrm{C} 8-\mathrm{C} 9-\mathrm{C} 10\left[-0.76(19)^{\circ}\right]$ and $\mathrm{C} 5-\mathrm{C} 6-\mathrm{C} 7-$ $\mathrm{O} 2\left[-1.18(19)^{\circ}\right]$ both in a syn-periplanar conformation. Those torsion angles result in an overall flat shape of the title compound with the dihedral angle between the terminal benzene rings being $9.18(6)^{\circ}$.

\section{Supramolecular features}

In the crystal, molecules are linked by $\mathrm{N}-\mathrm{H} \cdots \mathrm{O}$ and $\mathrm{O}-$ $\mathrm{H}$... O hydrogen bonds (Table 1) into a three-dimensional network. Molecules are connected into infinite chains along [101] through an $\mathrm{O} 4-\mathrm{H} 1 \mathrm{O} 4 \cdots \mathrm{O} 1^{\mathrm{iii}}$ hydrogen bond and those chains are further connected into two-dimensional plates parallel to the ac plane via $\mathrm{N} 1-\mathrm{H} 1 N 1 \cdots \mathrm{O} 3^{\mathrm{i}}$ and $\mathrm{O} 1-$ $\mathrm{H} 1 O 1 \cdots \mathrm{O} 2^{\mathrm{i}}$ hydrogen bonds with an $R_{2}^{2}(18)$ ring motif (Fig. $2 a$ and $3 a$; symmetry codes as in Table 1 ). Those plate are interconnected via an $\mathrm{O} 3-\mathrm{H} 1 \mathrm{O} 3 \cdots \mathrm{O} 2^{\mathrm{ii}}$ hydrogen bond with an $R_{2}^{2}(20)$ ring motif, forming a three-dimensional network (Fig. $2 b$ and $3 b$; symmetry code as in Table 1 ). In addition, the molecules are further stabilized by $\pi-\pi$ interactions involving

(a)
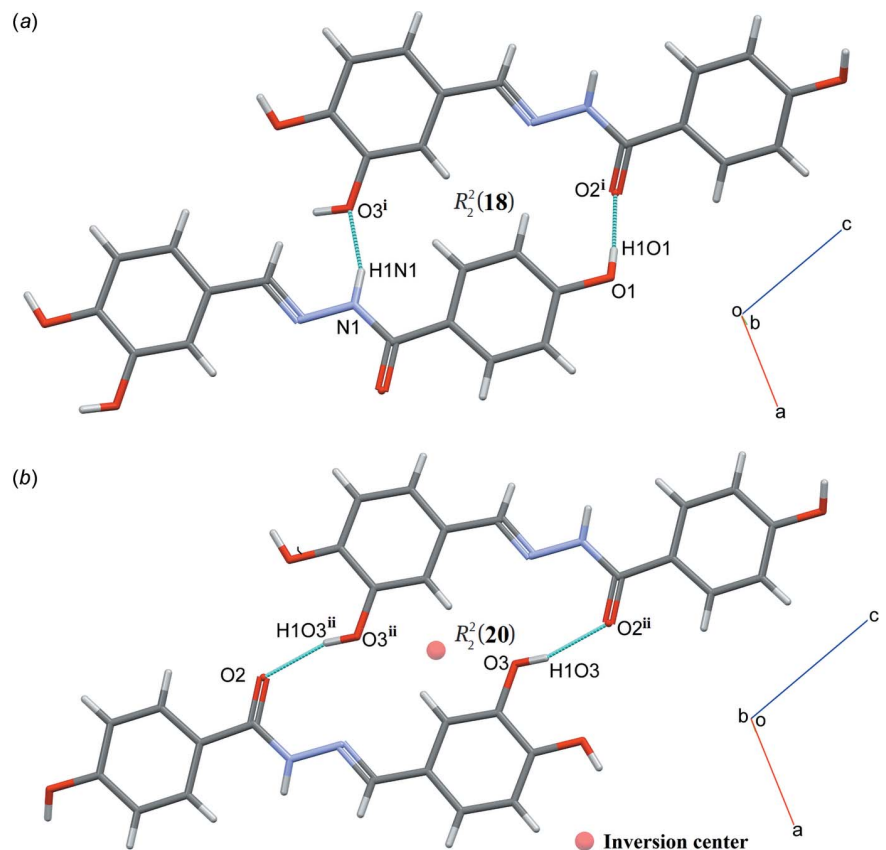

Figure 3

A view of dimers with $(a) R_{2}^{2}(18)$ and $(b) R_{2}^{2}(20)$ ring motifs. [Symmetry codes: (i) $x,-y+\frac{1}{2}, z+\frac{1}{2}$; (ii) $-x+1,-y+1,-z$.] 
Table 2

Selected torsion angles $\left(^{\circ}\right)$ and the dihedral angle $\left(^{\circ}\right)$ between the terminal benzene rings.

\begin{tabular}{|c|c|c|c|c|c|c|}
\hline Compound & $\boldsymbol{R}$ & $\tau_{1}$ & $\tau_{2}$ & $\tau_{3}$ & $\tau_{4}$ & Dihedral angle \\
\hline \multicolumn{7}{|l|}{ Planar } \\
\hline (I) & 3,4-dihydroxyphenyl & -1.2 & -177.3 & -175.0 & -0.8 & 9.2 \\
\hline ABALIA (Fun et al., 2011) & 3-hydroxy-4-methoxyphenyl & 3.2 & 178.4 & 170.1 & -14.2 & 24.2 \\
\hline CECZOB (Subashini et al., 2012) & 4-chlorophenyl & 26.1 & -174.4 & 166.6 & -8.9 & 5.8 \\
\hline CECZUH (Subashini et al., 2012) & 4-bromophenyl & 25.6 & -174.9 & 169.0 & -7.2 & 9.8 \\
\hline ESOTUD (Chantrapromma et al., 2016) & 3-methoxyphenyl & $-19.4,20.7$ & $-173.5,-177.8$ & $-175.7,-173.0$ & $1.2,0.6$ & $24.0,29.3$ \\
\hline HOZBII (Li \& Ban, 2009) & 4-nitrophenyl & 2.0 & 177.7 & 178.3 & -0.6 & 2.5 \\
\hline IJUKEE (Zhang, 2011) & 4-hydroxy-3-nitrophenyl & -7.2 & -177.0 & -179.3 & 6.0 & 5.5 \\
\hline IRAXEF (Sánchez-Lozano et al., 2011) & 2,4-dihydroxyphenyl & -7.7 & -177.8 & -177.2 & -4.1 & 6.9 \\
\hline MOZPEX (Ren, 2009) & 3,5-dichloro-2-hydroxyphenyl & 12.3 & 178.7 & -179.4 & -7.3 & 5.1 \\
\hline ROFMOP (Xue et al., 2008) & 3-bromo-5-chloro-2-hydroxyphenyl & -2.3 & 175.9 & -176.5 & -1.3 & 3.0 \\
\hline TEWLAL (Ayyannan et al., 2016) & 5-bromo-2-hydroxyphenyl & -15.7 & -173.6 & 168.9 & 3.1 & 27.0 \\
\hline WACVON (Shalash et al., 2010) & 4-hydroxy-3-methoxyphenyl & -34.2 & -175.5 & 174.7 & 15.4 & 28.6 \\
\hline WACXOP (Huang, 2010) & 2,4-dichlorophenyl & -14.3 & -179.8 & -175.0 & 3.0 & 7.0 \\
\hline YAGYAI (Horkaew et al., 2011) & 3,4,5-trimethoxyphenyl & -10.6 & 172.2 & 175.8 & 2.8 & 19.4 \\
\hline YIFPAF (Salhin et al., 2007) & 2-hydroxyphenyl & 18.8 & 179.5 & 178.7 & 3.3 & 21.7 \\
\hline ZAPKOS (Hou, 2012) & 3-nitrophenyl & -14.6 & 169.4 & 177.4 & 13.8 & 9.2 \\
\hline ZIPLAO (Prachumrat et al., 2018) & 2,3-dimethoxyphenyl & 9.6 & -175.3 & 172.9 & -1.3 & 9.3 \\
\hline \multicolumn{7}{|l|}{ Non-planar } \\
\hline CABWUA (Meng et al., 2014) & 2-hydroxy-5-methylphenyl & 18.4 & -178.5 & -169.8 & 8.0 & 40.8 \\
\hline CEDBAQ (Subashini et al., 2012) & 4-(diethylamino)phenyl & 34.9 & -178.5 & -151.7 & 8.75 & 77.3 \\
\hline HUCVIL (Hao, 2009) & 2-chlorophenyl & -22.5 & -179.2 & 177.4 & -4.2 & 30.5 \\
\hline HUCWOS (Shi, 2009) & 4-methoxyphenyl & -50.9 & -177.5 & 174.8 & 9.2 & 46.6 \\
\hline MOSPEQ (Qiu, 2009) & 5-chloro-2-hydroxyphenyl & 19.0 & -178.5 & -170.9 & 7.59 & 40.2 \\
\hline PAQJID (Gopal Reddy et al., 2017) & 4-ethylphenyl & -39.9 & 171.1 & 173.9 & 7.4 & 49.9 \\
\hline PAWVUG (Rassem et al., 2012a) & 2-methoxyphenyl & 29.1 & -166.8 & -175.1 & 19.2 & 66.6 \\
\hline PEDGOW (Saad et al., 2012) & 3-chlorophenyl & -21.1 & 179.5 & 175.3 & -9.3 & 39.0 \\
\hline XEBYUA (Rassem et al., 2012b) & 2-hydroxy-4-methoxyphenyl & 28.7 & 178.1 & -169.8 & 1.3 & 40.6 \\
\hline
\end{tabular}

both aromatic rings with $C g 1 \cdots C g 2^{\text {iv }}=3.6480(8) \AA$ and $C g 1 \cdots C g 2^{\mathrm{v}}=3.7607$ (8) $\AA$ [symmetry codes: (iv) $1-x,-\frac{1}{2}+y$, $\frac{1}{2}-z ;(\mathrm{v}) 1-x, \frac{1}{2}+y, \frac{1}{2}-z ; C g 1$ and $C g 2$ are the centroids of the $\mathrm{C} 1-\mathrm{C} 6$ and $\mathrm{C} 9-\mathrm{C} 14$ aromatic rings, respectively.]

\section{Database survey}

A search of the Cambridge Structural Database (CSD version 5.40, last update May 2019; Groom et al., 2016) using (E)-N'benzenylidene-4-hydroxybenzohydrazide as the reference moiety resulted in 31 structures with different substituents at the benzylidenyl ring. The different substituent $(\boldsymbol{R})$ together with selected torsion angles, $\tau_{1}(\mathrm{C} 5-\mathrm{C} 6-\mathrm{C} 7-\mathrm{O} 2), \tau_{2}(\mathrm{C} 6-$ $\mathrm{C} 7-\mathrm{N} 1-\mathrm{N} 2), \tau_{3}(\mathrm{C} 7-\mathrm{N} 1-\mathrm{N} 2-\mathrm{C} 8)$ and $\tau_{4}(\mathrm{~N} 2-\mathrm{C} 8-\mathrm{C} 9-$ $\mathrm{C} 10)$ as shown in Fig. 4, and the dihedral angle between the terminal aromatic rings are summarized in Table 2. The torsion angles $\tau_{2}$ and $\tau_{3}$ are anti-periplanar (151.7-179.8 $8^{\circ}$, showing that the hydrazide connecting bridges are nearly planar. As for the torsion angle $\tau_{4}$, all structures adopt a synperiplanar conformation $\left(0.6-19.6^{\circ}\right)$. Similar to the title compound, the $\tau_{1}$ torsion angles for most of the structures are syn-periplanar $\left(2.0-29.1^{\circ}\right)$. However, there are three outliers (CEDBAQ, HUCWOS and PAQJID) whose $\tau_{1}$ torsion angles are syn-clinal $\left(34.9-50.9^{\circ}\right)$. By comparing the dihedral angles, the structures can be divided into planar compounds (dihedral angle $=2.5-29.3^{\circ}$ ) and non-planar compounds (dihedral angle $\left.=30.5-77 \cdot 3^{\circ}\right)$. In general, as the hydrazide-connecting bridges are nearly planar, relatively flat $\tau_{1}$ and $\tau_{4}$ torsion angles are observed in the former compounds, while relatively twisted $\tau_{1}$ and $\tau_{4}$ torsion angles are observed in the latter.

\section{Synthesis and crystallization}

The title compound (I) was prepared by dissolving 4-hydroxybenzohydrazide $(2 \mathrm{mmol}, 0.30 \mathrm{~g})$ in ethanol $(10 \mathrm{ml})$. A solution of 3,4-dihydroxybenzaldehyde $(2 \mathrm{mmol}, 0.28 \mathrm{~g})$ in ethanol $(10 \mathrm{ml})$ was then added to the reaction. The mixture was refluxed for $6 \mathrm{~h}$ and the white solid of the product that appeared was collected by filtration, washed with ethanol and dried in air. Colourless single crystals of (I) were obtained after recrystallization from methanol at room temperature for several days.

M.p. 572-573 K. UV-Vis (MeOH) $\lambda_{\max } 213,327 \mathrm{~nm}$; FT-IR $(\mathrm{KBr}) v\left(\mathrm{~cm}^{-1}\right): 3121(\mathrm{O}-\mathrm{H}$ stretching $), 2800(\mathrm{C}-\mathrm{H}$ aromatic stretching), 1615 (amide $\mathrm{C}=\mathrm{O}$ stretching), $1570(\mathrm{C}=\mathrm{N}$ stretching), $1506(\mathrm{C}=\mathrm{C}$ stretching of aromatic compound) $\mathrm{cm}^{-1}$; ${ }^{1} \mathrm{H}$ NMR (300 MHz, $d_{6}$-DMSO) $\delta 11.39(s, 1 \mathrm{H}, \mathrm{NH})$, $10.10(s, 1 \mathrm{H}, \mathrm{Ar}-\mathrm{OH}), 8.23(s, 1 \mathrm{H}, \mathrm{N}=\mathrm{CH}), 7.77(d, J=$

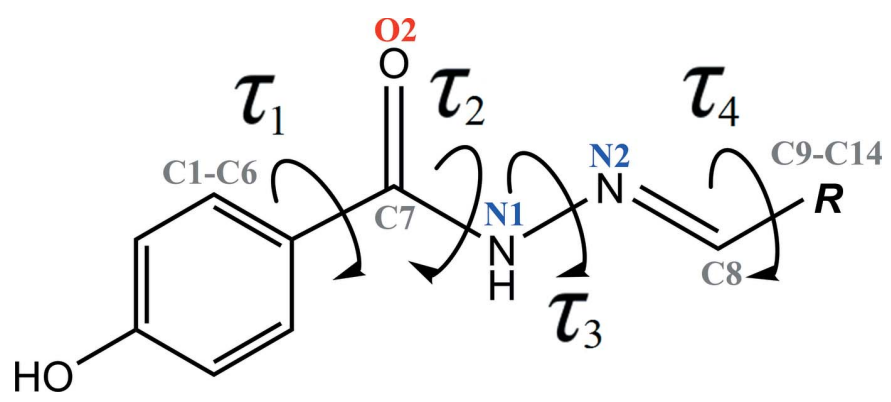

Figure 4

General chemical diagram, showing torsion angles $\tau_{1}, \tau_{2}, \tau_{3}$ and $\tau_{4}$ in the benzylidene-4-hydroxybenzohydrazide derivative. 
Table 3

Experimental details.

\begin{tabular}{|c|c|}
\hline \multicolumn{2}{|l|}{ Crystal data } \\
\hline Chemical formula & $\mathrm{C}_{14} \mathrm{H}_{12} \mathrm{~N}_{2} \mathrm{O}_{4}$ \\
\hline$M_{\mathrm{r}}$ & 272.26 \\
\hline Crystal system, space group & Monoclinic, $P 2_{1} / c$ \\
\hline Temperature (K) & 296 \\
\hline$a, b, c(\AA)$ & $\begin{array}{l}11.5352(8), 7.1711(5), \\
\quad 15.0606(10)\end{array}$ \\
\hline$\beta\left({ }^{\circ}\right)$ & $108.548(2)$ \\
\hline$V\left(\mathrm{~A}^{3}\right)$ & $1181.10(14)$ \\
\hline$Z$ & 4 \\
\hline Radiation type & Мо $K \alpha$ \\
\hline$\mu\left(\mathrm{mm}^{-1}\right)$ & 0.11 \\
\hline Crystal size $(\mathrm{mm})$ & $0.80 \times 0.21 \times 0.07$ \\
\hline \multicolumn{2}{|l|}{ Data collection } \\
\hline Diffractometer & $\begin{array}{l}\text { Bruker APEXII DUO CCD area- } \\
\text { detector }\end{array}$ \\
\hline Absorption correction & $\begin{array}{l}\text { Multi-scan (SADABS; Bruker, } \\
\text { 2012) }\end{array}$ \\
\hline$T_{\min }, T_{\max }$ & $0.924,0.954$ \\
\hline $\begin{array}{l}\text { No. of measured, independent and } \\
\text { observed }[I>2 \sigma(I)] \text { reflections }\end{array}$ & $22559,3200,2453$ \\
\hline$R_{\text {int }}$ & 0.024 \\
\hline$(\sin \theta / \lambda)_{\max }\left(\AA^{-1}\right)$ & 0.686 \\
\hline \multicolumn{2}{|l|}{ Refinement } \\
\hline$R\left[F^{2}>2 \sigma\left(F^{2}\right)\right], w R\left(F^{2}\right), S$ & $0.050,0.161,1.05$ \\
\hline No. of reflections & 3200 \\
\hline No. of parameters & 197 \\
\hline $\mathrm{H}$-atom treatment & $\begin{array}{l}\mathrm{H} \text { atoms treated by a mixture of } \\
\text { independent and constrained } \\
\text { refinement }\end{array}$ \\
\hline$\Delta \rho_{\max }, \Delta \rho_{\min }\left(\mathrm{e} \AA^{-3}\right)$ & $0.35,-0.19$ \\
\hline
\end{tabular}

Computer programs: APEX2 and SAINT (Bruker, 2012), SHELXS97 (Sheldrick, 2008), SHELXL2013 (Sheldrick, 2015), Mercury (Macrae et al., 2006) and PLATON (Spek, 2009).

$8.4 \mathrm{~Hz}, 2 \mathrm{H}, \mathrm{Ar}-\mathrm{H}), 6.84(d, J=8.4,2 \mathrm{H}, \mathrm{Ar}-\mathrm{H}), 9.33(s, 2 \mathrm{H}$, $\mathrm{Ar}-\mathrm{OH}), 7.22(s, 1 \mathrm{H}, \mathrm{Ar}-\mathrm{H}), 6.90(d, J=7.8 \mathrm{~Hz}, 1 \mathrm{H}, \mathrm{Ar}-$ $\mathrm{H}), 6.77(d, J=8.1 \mathrm{~Hz}, 1 \mathrm{H}, \mathrm{Ar}-\mathrm{H})$.

\section{Refinement}

Crystal data, data collection and structure refinement details are summarized in Table 3. C-bound $\mathrm{H}$ atoms were positioned geometrically $(\mathrm{C}-\mathrm{H}=0.93 \AA)$ and refined using a riding model with $U_{\text {iso }}(\mathrm{H})=1.2 U_{\text {eq }}(\mathrm{C})$. All O- and $\mathrm{N}$-bound $\mathrm{H}$ atoms were located in a difference-Fourier map and refined freely $[\mathrm{O}-\mathrm{H}=0.80(2)-0.88(2) \AA$ and $\mathrm{N}-\mathrm{H}=0.87$ (2) $\AA]$.

\section{Acknowledgements}

The authors thank Prince of Songkla University for research grant (SCI590716S). PP thanks the Graduate School, Prince of Songkla University for partial financial support. The authors extend their appreciation to the Universiti Sains Malaysia for the research facilities.

\section{Funding information}

The authors thank Prince of Songkla University for research grant (SCI590716S). PP thanks the Graduate School, Prince of Songkla University for partial financial support.

\section{References}

Ayyannan, G., Mohanraj, M., Raja, G., Bhuvanesh, N., Nandhakumar, R. \& Jayabalakrishnan, C. (2016). J. Photochem. Photobiol. B, 163, $1-13$.

Aziz, A. N., Taha, M., Ismail, N. H., Anouar, el H., Yousuf, S., Jamil, W., Awang, K., Ahmat, N., Khan, K. M. \& Kashif, S. M. (2014). Molecules, 19, 8414-8433.

Bachhawat, A., Shihabudeen, J. M. S. \& Thirumurugan, K. (2011). Int. J. Pharm. Pharm. Sci. 3, 267-274.

Bruker (2012). APEX2, SAINT and SADABS. Bruker AXS Inc., Madison. Wisconsin, USA.

Chantrapromma, S., Prachumrat, P., Ruanwas, P., Boonnak, N. \& Kassim, M. B. (2016). Acta Cryst. E72, 1339-1342.

Fun, H.-K., Horkaew, J. \& Chantrapromma, S. (2011). Acta Cryst. E67, o2644-o2645.

Gopal Reddy, N. B., Krishna, P. M., Shantha Kumar, S. S., Patil, Y. P. \& Nethaji, M. (2017). J. Mol. Struct. 1137, 543-552.

Groom, C. R., Bruno, I. J., Lightfoot, M. P. \& Ward, S. C. (2016). Acta Cryst. B72, 171-179.

Hao, Y.-M. (2009). Acta Cryst. E65, o2098.

Horkaew, J., Chantrapromma, S. \& Fun, H.-K. (2011). Acta Cryst. E67, o2985.

Hou, J.-L. (2012). Acta Cryst. E68, o1352.

Huang, H.-W. (2010). Acta Cryst. E66, o3143.

Kumari, D. \& Bansal, H. (2018). Pharm. Innov. J, 7, 543-550.

Li, C.-M. \& Ban, H.-Y. (2009). Acta Cryst. E65, o1466.

Macrae, C. F., Edgington, P. R., McCabe, P., Pidcock, E., Shields, G. P., Taylor, R., Towler, M. \& van de Streek, J. (2006). J. Appl. Cryst. 39, 453-457.

Maheswari, R. \& Manjula, J. (2015). Inter. J. Applied Res. 1, 587-592.

Meng, X.-F., Li, W.-N. \& Ma, J.-J. (2014). J. Chil. Chem. Soc. 59, $2647-$ 2651.

Narasimhan, B., Kumar, P. \& Sharma, D. (2010). Acta Pharm. Sci. 52, 169-180.

Prachumrat, P., Kobkeatthawin, T., Ruanwas, P., Boonnak, N., Laphookhieo, S., Kassim, M. B. \& Chantrapromma, S. (2018). Crystallogr. Rep. 63, 405-411.

Qiu, X.-Y. (2009). Acta Cryst. E65, o975.

Rassem, H. H., Salhin, A., Bin Salleh, B., Rosli, M. M. \& Fun, H.-K. (2012a). Acta Cryst. E68, o1832.

Rassem, H. H., Salhin, A., Bin Salleh, B., Rosli, M. M. \& Fun, H.-K. (2012b). Acta Cryst. E68, o2279.

Ren, C.-G. (2009). Acta Cryst. E65, o1503-01504.

Saad, S. M., Fatima, I., Perveen, S., Khan, K. M. \& Yousuf, S. (2012). Acta Cryst. E68, o3499.

Salhin, A., Tameem, A. A., Saad, B., Ng, S.-L. \& Fun, H.-K. (2007). Acta Cryst. E63, o2880.

Sánchez-Lozano, M., Vázquez-López, E. M., Hermida-Ramón, J. M. \& Estévez, C. M. (2011). Polyhedron, 30, 953-962.

Shalash, M., Salhin, A., Adnan, R., Yeap, C. S. \& Fun, H.-K. (2010). Acta Cryst. E66, o3126-03127.

Sheldrick, G. M. (2008). Acta Cryst. A64, 112-122.

Sheldrick, G. M. (2015). Acta Cryst. C71, 3-8.

Shi, D.-H. (2009). Acta Cryst. E65, o2107.

Spek, A. L. (2009). Acta Cryst. D65, 148-155.

Subashini, A., Ramamurthi, K. \& Stoeckli-Evans, H. (2012). Acta Cryst. C68, o408-0412.

Taha, M., Ismail, N. H., Imran, S., Rokei, M. Q., Saad, S. M. \& Khan, K. M. (2015). Bioorg. Med. Chem. 23, 4155-4162.

Thaipong, K., Boonprakob, U., Crosby, K., Cisneros-Zevallos, L. \& Byrne, D. H. (2006). J. Food. Composition Anal. 19, 669-675.

Xia, Z., Hu, L. W. \& Wang, X. (2007). Bioorg. Med. Chem. 17, 33743377.

Xue, L.-W., Han, Y.-J., Hao, C.-J., Zhao, G.-Q. \& Liu, Q.-R. (2008). Acta Cryst. E64, o1938.

Zhang, Z. (2011). Acta Cryst. E67, o300. 


\section{supporting information}

Acta Cryst. (2019). E75, 1280-1283 [https://doi.org/10.1107/S2056989019010442]

Crystal structure of (E)-N'-(3,4-dihydroxybenzylidene)-4-hydroxybenzohydrazide

Suchada Chantrapromma, Huey Chong Kwong, Patcharawadee Prachumrat, Thawanrat

Kobkeatthawin, Tze Shyang Chia and Ching Kheng Quah

Computing details

Data collection: APEX2 (Bruker, 2012); cell refinement: SAINT (Bruker, 2012); data reduction: SAINT (Bruker, 2012); program(s) used to solve structure: SHELXS97 (Sheldrick, 2008); program(s) used to refine structure: SHELXL2013 (Sheldrick, 2015); molecular graphics: SHELXL2013 (Sheldrick, 2015) and Mercury (Macrae et al., 2006); software used to prepare material for publication: SHELXL2013 (Sheldrick, 2015) and PLATON (Spek, 2009).

(E)-N'-(3,4-Dihydroxybenzylidene)-4-hydroxybenzohydrazide

Crystal data

$\mathrm{C}_{14} \mathrm{H}_{12} \mathrm{~N}_{2} \mathrm{O}_{4}$

$M_{r}=272.26$

Monoclinic, $P 2_{1} / c$

$a=11.5352(8) \AA$

$b=7.1711(5) \AA$

$c=15.0606(10) \AA$

$\beta=108.548(2)^{\circ}$

$V=1181.10(14) \AA^{3}$

$Z=4$

\section{Data collection}

Bruker APEXII DUO CCD area-detector diffractometer

Radiation source: fine-focus sealed tube Graphite monochromator $\varphi$ and $\omega$ scans

Absorption correction: multi-scan

(SADABS; Bruker, 2012)

$T_{\min }=0.924, T_{\max }=0.954$

\section{Refinement}

Refinement on $F^{2}$

Least-squares matrix: full

$R\left[F^{2}>2 \sigma\left(F^{2}\right)\right]=0.050$

$w R\left(F^{2}\right)=0.161$

$S=1.05$

3200 reflections

197 parameters

0 restraints
$F(000)=568$

$D_{\mathrm{x}}=1.531 \mathrm{Mg} \mathrm{m}^{-3}$

Mo $K \alpha$ radiation, $\lambda=0.71073 \AA$

Cell parameters from 6293 reflections

$\theta=2.9-29.2^{\circ}$

$\mu=0.11 \mathrm{~mm}^{-1}$

$T=296 \mathrm{~K}$

Plate, colourless

$0.80 \times 0.21 \times 0.07 \mathrm{~mm}$

22559 measured reflections

3200 independent reflections

2453 reflections with $I>2 \sigma(I)$

$R_{\text {int }}=0.024$

$\theta_{\max }=29.2^{\circ}, \theta_{\min }=1.9^{\circ}$

$h=-15 \rightarrow 15$

$k=-9 \rightarrow 9$

$l=-20 \rightarrow 20$

Hydrogen site location: mixed

$\mathrm{H}$ atoms treated by a mixture of independent and constrained refinement

$w=1 /\left[\sigma^{2}\left(F_{\mathrm{o}}^{2}\right)+(0.0999 P)^{2}+0.1317 P\right]$

where $P=\left(F_{\mathrm{o}}^{2}+2 F_{\mathrm{c}}^{2}\right) / 3$

$(\Delta / \sigma)_{\max }<0.001$

$\Delta \rho_{\max }=0.35$ e $\AA^{-3}$

$\Delta \rho_{\min }=-0.18$ e $\AA^{-3}$ 


\section{Special details}

Geometry. All esds (except the esd in the dihedral angle between two 1.s. planes) are estimated using the full covariance matrix. The cell esds are taken into account individually in the estimation of esds in distances, angles and torsion angles; correlations between esds in cell parameters are only used when they are defined by crystal symmetry. An approximate (isotropic) treatment of cell esds is used for estimating esds involving l.s. planes.

Fractional atomic coordinates and isotropic or equivalent isotropic displacement parameters $\left(\AA^{2}\right)$

\begin{tabular}{lllll}
\hline & $x$ & $y$ & $z$ & $U_{\text {iso }} / U_{\text {eq }}$ \\
\hline O1 & $0.87093(9)$ & $0.31797(18)$ & $0.75119(6)$ & $0.0493(3)$ \\
O2 & $0.72990(8)$ & $0.42338(15)$ & $0.31133(6)$ & $0.0439(3)$ \\
O3 & $0.36583(9)$ & $0.37514(15)$ & $-0.12560(6)$ & $0.0432(3)$ \\
O4 & $0.11997(10)$ & $0.35694(18)$ & $-0.18715(7)$ & $0.0547(3)$ \\
N1 & $0.54178(10)$ & $0.37659(17)$ & $0.32223(7)$ & $0.0392(3)$ \\
N2 & $0.48862(10)$ & $0.38068(17)$ & $0.22612(7)$ & $0.0387(3)$ \\
C1 & $0.64041(11)$ & $0.3493(2)$ & $0.52044(8)$ & $0.0360(3)$ \\
H1A & 0.555998 & 0.341901 & 0.493279 & $0.043^{*}$ \\
C2 & $0.69125(11)$ & $0.32969(19)$ & $0.61626(8)$ & $0.0370(3)$ \\
H2A & 0.641478 & 0.308993 & 0.653221 & $0.044^{*}$ \\
C3 & $0.81673(12)$ & $0.34105(19)$ & $0.65681(8)$ & $0.0359(3)$ \\
C4 & $0.89049(12)$ & $0.3773(2)$ & $0.60204(9)$ & $0.0429(3)$ \\
H4A & 0.974622 & 0.389056 & 0.629625 & $0.051^{*}$ \\
C5 & $0.83860(12)$ & $0.3960(2)$ & $0.50639(9)$ & $0.0398(3)$ \\
H5A & 0.888474 & 0.419686 & 0.469812 & $0.048^{*}$ \\
C6 & $0.71320(11)$ & $0.38002(16)$ & $0.46381(8)$ & $0.0312(3)$ \\
C7 & $0.66306(11)$ & $0.39454(18)$ & $0.36014(8)$ & $0.0334(3)$ \\
C8 & $0.37218(12)$ & $0.37651(18)$ & $0.19709(8)$ & $0.0357(3)$ \\
H8A & 0.329660 & 0.374477 & 0.240199 & $0.043^{*}$ \\
C9 & $0.30468(12)$ & $0.37489(17)$ & $0.09713(8)$ & $0.0332(3)$ \\
C10 & $0.36617(11)$ & $0.38007(18)$ & $0.03124(8)$ & $0.0339(3)$ \\
H10A & 0.451058 & 0.387612 & 0.051040 & $0.041^{*}$ \\
C11 & $0.30251(11)$ & $0.37414(17)$ & $-0.06263(8)$ & $0.0332(3)$ \\
C12 & $0.17505(12)$ & $0.36368(19)$ & $-0.09316(9)$ & $0.0377(3)$ \\
C13 & $0.11375(12)$ & $0.3624(2)$ & $-0.02790(9)$ & $0.0434(3)$ \\
H13A & 0.028756 & 0.358471 & -0.047790 & $0.052^{*}$ \\
C14 & $0.17809(12)$ & $0.3669(2)$ & $0.06705(9)$ & $0.0400(3)$ \\
H14A & 0.136252 & 0.364586 & 0.110600 & $0.048^{*}$ \\
H1O1 & $0.8288(18)$ & $0.256(3)$ & $0.7732(15)$ & $0.074(6)^{*}$ \\
H1O3 & $0.324(2)$ & $0.421(3)$ & $-0.1808(16)$ & $0.092(7)^{*}$ \\
H1O4 & $0.046(2)$ & $0.345(3)$ & $-0.1964(16)$ & $0.085(7)^{*}$ \\
H1N1 & $0.4954(19)$ & $0.342(3)$ & $0.3546(14)$ & $0.069(6)^{*}$ \\
& & & &
\end{tabular}

Atomic displacement parameters $\left(\AA^{2}\right)$

\begin{tabular}{lllllll}
\hline & $U^{11}$ & $U^{22}$ & $U^{33}$ & $U^{12}$ & $U^{13}$ & $U^{23}$ \\
\hline O1 & $0.0364(5)$ & $0.0836(8)$ & $0.0215(4)$ & $-0.0105(5)$ & $0.0004(4)$ & $0.0057(4)$ \\
O2 & $0.0376(5)$ & $0.0700(7)$ & $0.0267(4)$ & $-0.0036(4)$ & $0.0139(4)$ & $-0.0016(4)$ \\
O3 & $0.0344(5)$ & $0.0719(7)$ & $0.0236(4)$ & $0.0037(4)$ & $0.0095(4)$ & $0.0026(4)$
\end{tabular}




\begin{tabular}{lllllll} 
O4 & $0.0352(6)$ & $0.0991(9)$ & $0.0227(5)$ & $-0.0083(5)$ & $-0.0009(4)$ & $0.0033(5)$ \\
N1 & $0.0326(6)$ & $0.0651(7)$ & $0.0185(5)$ & $-0.0038(5)$ & $0.0060(4)$ & $0.0023(4)$ \\
N2 & $0.0380(6)$ & $0.0576(7)$ & $0.0182(5)$ & $-0.0033(5)$ & $0.0056(4)$ & $0.0010(4)$ \\
C1 & $0.0254(6)$ & $0.0554(7)$ & $0.0248(6)$ & $-0.0019(5)$ & $0.0046(4)$ & $-0.0004(5)$ \\
C2 & $0.0319(6)$ & $0.0558(8)$ & $0.0232(6)$ & $-0.0014(5)$ & $0.0086(5)$ & $0.0005(5)$ \\
C3 & $0.0335(6)$ & $0.0485(7)$ & $0.0220(5)$ & $-0.0024(5)$ & $0.0037(5)$ & $-0.0001(5)$ \\
C4 & $0.0272(6)$ & $0.0688(9)$ & $0.0289(6)$ & $-0.0039(6)$ & $0.0037(5)$ & $0.0022(6)$ \\
C5 & $0.0307(6)$ & $0.0611(8)$ & $0.0284(6)$ & $-0.0029(5)$ & $0.0105(5)$ & $0.0014(5)$ \\
C6 & $0.0300(6)$ & $0.0411(6)$ & $0.0213(5)$ & $0.0007(4)$ & $0.0063(4)$ & $-0.0010(4)$ \\
C7 & $0.0337(6)$ & $0.0440(7)$ & $0.0222(6)$ & $-0.0001(5)$ & $0.0086(5)$ & $-0.0019(4)$ \\
C8 & $0.0352(7)$ & $0.0481(7)$ & $0.0231(6)$ & $0.0003(5)$ & $0.0081(5)$ & $0.0012(5)$ \\
C9 & $0.0332(6)$ & $0.0416(6)$ & $0.0219(5)$ & $0.0005(5)$ & $0.0046(5)$ & $0.0009(4)$ \\
C10 & $0.0261(5)$ & $0.0492(7)$ & $0.0234(6)$ & $0.0004(5)$ & $0.0038(4)$ & $-0.0001(5)$ \\
C11 & $0.0311(6)$ & $0.0443(7)$ & $0.0234(5)$ & $0.0008(5)$ & $0.0073(4)$ & $0.0010(4)$ \\
C12 & $0.0313(6)$ & $0.0521(7)$ & $0.0243(6)$ & $-0.0013(5)$ & $0.0012(5)$ & $0.0026(5)$ \\
C13 & $0.0255(6)$ & $0.0686(9)$ & $0.0321(7)$ & $-0.0003(5)$ & $0.0038(5)$ & $0.0046(6)$ \\
C14 & $0.0329(6)$ & $0.0582(8)$ & $0.0298(6)$ & $0.0015(5)$ & $0.0110(5)$ & $0.0037(5)$ \\
& & & & & & \\
\hline
\end{tabular}

Geometric parameters $\left(A,{ }^{\circ}\right)$

\begin{tabular}{llll}
\hline $\mathrm{O} 1-\mathrm{C} 3$ & $1.3688(14)$ & $\mathrm{C} 4-\mathrm{C} 5$ & $1.3794(18)$ \\
$\mathrm{O} 1-\mathrm{H} 1 \mathrm{O} 1$ & $0.80(2)$ & $\mathrm{C}-\mathrm{H} 4 \mathrm{~A}$ & 0.9300 \\
$\mathrm{O} 2-\mathrm{C} 7$ & $1.2403(15)$ & $\mathrm{C} 5-\mathrm{C} 6$ & $1.3880(18)$ \\
$\mathrm{O} 3-\mathrm{C} 11$ & $1.3688(15)$ & $\mathrm{C} 5-\mathrm{H} 5 \mathrm{~A}$ & 0.9300 \\
$\mathrm{O} 3-\mathrm{H} 1 \mathrm{O} 3$ & $0.88(2)$ & $\mathrm{C} 6-\mathrm{C} 7$ & $1.4861(15)$ \\
$\mathrm{O} 4-\mathrm{C} 12$ & $1.3556(15)$ & $\mathrm{C} 8-\mathrm{C} 9$ & $1.4584(16)$ \\
$\mathrm{O} 4-\mathrm{H} 1 \mathrm{O} 4$ & $0.83(3)$ & $\mathrm{C} 8-\mathrm{H} 8 \mathrm{~A}$ & 0.9300 \\
$\mathrm{~N} 1-\mathrm{C} 7$ & $1.3385(17)$ & $\mathrm{C} 9-\mathrm{C} 14$ & $1.3856(18)$ \\
$\mathrm{N} 1-\mathrm{N} 2$ & $1.3811(14)$ & $\mathrm{C} 9-\mathrm{C} 10$ & $1.3918(17)$ \\
$\mathrm{N} 1-\mathrm{H} 1 \mathrm{~N} 1$ & $0.87(2)$ & $\mathrm{C} 10-\mathrm{C} 11$ & $1.3709(16)$ \\
$\mathrm{N} 2-\mathrm{C} 8$ & $1.2738(17)$ & $\mathrm{C} 10-\mathrm{H} 10 \mathrm{~A}$ & 0.9300 \\
$\mathrm{C} 1-\mathrm{C} 2$ & $1.3812(16)$ & $\mathrm{C} 11-\mathrm{C} 12$ & $1.3959(18)$ \\
$\mathrm{C} 1-\mathrm{C} 6$ & $1.3916(17)$ & $\mathrm{C} 12-\mathrm{C} 13$ & $1.3815(19)$ \\
$\mathrm{C} 1-\mathrm{H} 1 \mathrm{~A}$ & 0.9300 & $\mathrm{C} 13-\mathrm{C} 14$ & $1.3861(17)$ \\
$\mathrm{C} 2-\mathrm{C} 3$ & $1.3828(18)$ & $\mathrm{C} 13-\mathrm{H} 13 \mathrm{~A}$ & 0.9300 \\
$\mathrm{C} 2-\mathrm{H} 2 \mathrm{~A}$ & 0.9300 & $\mathrm{C} 14-\mathrm{H} 14 \mathrm{~A}$ & \\
$\mathrm{C} 3-\mathrm{C} 4$ & $1.3852(19)$ & & $121.71(11)$ \\
& & & $121.79(11)$ \\
$\mathrm{C} 3-\mathrm{O} 1-\mathrm{H} 1 \mathrm{O} 1$ & $\mathrm{O} 2-\mathrm{C} 7-\mathrm{N} 1$ & $116.49(11)$ \\
$\mathrm{C} 11-\mathrm{O} 3-\mathrm{H} 1 \mathrm{O} 3$ & $111.0(15)$ & $\mathrm{O} 2-\mathrm{C} 7-\mathrm{C} 6$ & $120.86(12)$ \\
$\mathrm{C} 12-\mathrm{O} 4-\mathrm{H} 1 \mathrm{O} 4$ & $113.5(15)$ & $\mathrm{N} 1-\mathrm{C} 7-\mathrm{C} 6$ & 119.6 \\
$\mathrm{C} 7-\mathrm{N} 1-\mathrm{N} 2$ & $107.2(16)$ & $\mathrm{N} 2-\mathrm{C} 8-\mathrm{C} 9$ & 119.6 \\
$\mathrm{C} 7-\mathrm{N} 1-\mathrm{H} 1 \mathrm{~N} 1$ & $120.02(11)$ & $\mathrm{N} 2-\mathrm{C} 8-\mathrm{H} 8 \mathrm{~A}$ & $119.41(11)$ \\
$\mathrm{N} 2-\mathrm{N} 1-\mathrm{H} 1 \mathrm{~N} 1$ & $122.4(13)$ & $\mathrm{C} 9-\mathrm{C} 8-\mathrm{H} 8 \mathrm{~A}$ & $119.93(12)$ \\
$\mathrm{C} 8-\mathrm{N} 2-\mathrm{N} 1$ & $116.6(13)$ & $\mathrm{C} 14-\mathrm{C} 9-\mathrm{C} 8$ & $120.66(11)$ \\
$\mathrm{C} 2-\mathrm{C} 1-\mathrm{C} 6$ & $115.30(11)$ & $\mathrm{C} 11-\mathrm{C} 10-\mathrm{C} 8$ & $120.47(11)$ \\
$\mathrm{C} 2-\mathrm{C} 1-\mathrm{H} 1 \mathrm{~A}$ & $121.20(11)$ & 119.4 &
\end{tabular}




\begin{tabular}{|c|c|c|c|}
\hline $\mathrm{C} 1-\mathrm{C} 2-\mathrm{C} 3$ & $119.49(11)$ & $\mathrm{C} 11-\mathrm{C} 10-\mathrm{H} 10 \mathrm{~A}$ & 119.8 \\
\hline $\mathrm{C} 1-\mathrm{C} 2-\mathrm{H} 2 \mathrm{~A}$ & 120.3 & $\mathrm{C} 9-\mathrm{C} 10-\mathrm{H} 10 \mathrm{~A}$ & 119.8 \\
\hline $\mathrm{C} 3-\mathrm{C} 2-\mathrm{H} 2 \mathrm{~A}$ & 120.3 & $\mathrm{O} 3-\mathrm{C} 11-\mathrm{C} 10$ & $119.04(11)$ \\
\hline $\mathrm{O} 1-\mathrm{C} 3-\mathrm{C} 2$ & $121.31(12)$ & $\mathrm{O} 3-\mathrm{C} 11-\mathrm{C} 12$ & $120.68(11)$ \\
\hline $\mathrm{O} 1-\mathrm{C} 3-\mathrm{C} 4$ & $118.49(11)$ & $\mathrm{C} 10-\mathrm{C} 11-\mathrm{C} 12$ & $120.26(11)$ \\
\hline $\mathrm{C} 2-\mathrm{C} 3-\mathrm{C} 4$ & $120.20(11)$ & $\mathrm{O} 4-\mathrm{C} 12-\mathrm{C} 13$ & $124.53(12)$ \\
\hline $\mathrm{C} 5-\mathrm{C} 4-\mathrm{C} 3$ & $119.69(12)$ & $\mathrm{O} 4-\mathrm{C} 12-\mathrm{C} 11$ & $116.14(12)$ \\
\hline $\mathrm{C} 5-\mathrm{C} 4-\mathrm{H} 4 \mathrm{~A}$ & 120.2 & $\mathrm{C} 13-\mathrm{C} 12-\mathrm{C} 11$ & $119.33(11)$ \\
\hline $\mathrm{C} 3-\mathrm{C} 4-\mathrm{H} 4 \mathrm{~A}$ & 120.2 & $\mathrm{C} 12-\mathrm{C} 13-\mathrm{C} 14$ & $120.43(12)$ \\
\hline $\mathrm{C} 4-\mathrm{C} 5-\mathrm{C} 6$ & $121.12(12)$ & $\mathrm{C} 12-\mathrm{C} 13-\mathrm{H} 13 \mathrm{~A}$ & 119.8 \\
\hline $\mathrm{C} 4-\mathrm{C} 5-\mathrm{H} 5 \mathrm{~A}$ & 119.4 & $\mathrm{C} 14-\mathrm{C} 13-\mathrm{H} 13 \mathrm{~A}$ & 119.8 \\
\hline $\mathrm{C} 6-\mathrm{C} 5-\mathrm{H} 5 \mathrm{~A}$ & 119.4 & $\mathrm{C} 9-\mathrm{C} 14-\mathrm{C} 13$ & $120.07(12)$ \\
\hline $\mathrm{C} 5-\mathrm{C} 6-\mathrm{C} 1$ & $118.24(11)$ & $\mathrm{C} 9-\mathrm{C} 14-\mathrm{H} 14 \mathrm{~A}$ & 120.0 \\
\hline $\mathrm{C} 5-\mathrm{C} 6-\mathrm{C} 7$ & $118.67(11)$ & $\mathrm{C} 13-\mathrm{C} 14-\mathrm{H} 14 \mathrm{~A}$ & 120.0 \\
\hline $\mathrm{C} 1-\mathrm{C} 6-\mathrm{C} 7$ & $123.09(11)$ & & \\
\hline $\mathrm{C} 7-\mathrm{N} 1-\mathrm{N} 2-\mathrm{C} 8$ & $-174.98(12)$ & $\mathrm{N} 1-\mathrm{N} 2-\mathrm{C} 8-\mathrm{C} 9$ & $-178.29(10)$ \\
\hline $\mathrm{C} 6-\mathrm{C} 1-\mathrm{C} 2-\mathrm{C} 3$ & $0.1(2)$ & $\mathrm{N} 2-\mathrm{C} 8-\mathrm{C} 9-\mathrm{C} 14$ & $178.83(12)$ \\
\hline $\mathrm{C} 1-\mathrm{C} 2-\mathrm{C} 3-\mathrm{O} 1$ & $-178.44(12)$ & $\mathrm{N} 2-\mathrm{C} 8-\mathrm{C} 9-\mathrm{C} 10$ & $-0.76(19)$ \\
\hline $\mathrm{C} 1-\mathrm{C} 2-\mathrm{C} 3-\mathrm{C} 4$ & $1.9(2)$ & $\mathrm{C} 14-\mathrm{C} 9-\mathrm{C} 10-\mathrm{C} 11$ & $-1.11(18)$ \\
\hline $\mathrm{O} 1-\mathrm{C} 3-\mathrm{C} 4-\mathrm{C} 5$ & $178.19(13)$ & $\mathrm{C} 8-\mathrm{C} 9-\mathrm{C} 10-\mathrm{C} 11$ & $178.47(11)$ \\
\hline $\mathrm{C} 2-\mathrm{C} 3-\mathrm{C} 4-\mathrm{C} 5$ & $-2.1(2)$ & $\mathrm{C} 9-\mathrm{C} 10-\mathrm{C} 11-\mathrm{O} 3$ & $-178.51(12)$ \\
\hline $\mathrm{C} 3-\mathrm{C} 4-\mathrm{C} 5-\mathrm{C} 6$ & $0.3(2)$ & $\mathrm{C} 9-\mathrm{C} 10-\mathrm{C} 11-\mathrm{C} 12$ & $0.29(19)$ \\
\hline $\mathrm{C} 4-\mathrm{C} 5-\mathrm{C} 6-\mathrm{C} 1$ & $1.6(2)$ & $\mathrm{O} 3-\mathrm{C} 11-\mathrm{C} 12-\mathrm{O} 4$ & $-0.71(19)$ \\
\hline $\mathrm{C} 4-\mathrm{C} 5-\mathrm{C} 6-\mathrm{C} 7$ & $-177.78(12)$ & $\mathrm{C} 10-\mathrm{C} 11-\mathrm{C} 12-\mathrm{O} 4$ & $-179.50(12)$ \\
\hline $\mathrm{C} 2-\mathrm{C} 1-\mathrm{C} 6-\mathrm{C} 5$ & $-1.9(2)$ & $\mathrm{O} 3-\mathrm{C} 11-\mathrm{C} 12-\mathrm{C} 13$ & $179.81(13)$ \\
\hline $\mathrm{C} 2-\mathrm{C} 1-\mathrm{C} 6-\mathrm{C} 7$ & $177.51(12)$ & $\mathrm{C} 10-\mathrm{C} 11-\mathrm{C} 12-\mathrm{C} 13$ & $1.03(19)$ \\
\hline $\mathrm{N} 2-\mathrm{N} 1-\mathrm{C} 7-\mathrm{O} 2$ & $3.3(2)$ & $\mathrm{O} 4-\mathrm{C} 12-\mathrm{C} 13-\mathrm{C} 14$ & $179.03(13)$ \\
\hline $\mathrm{N} 2-\mathrm{N} 1-\mathrm{C} 7-\mathrm{C} 6$ & $-177.33(10)$ & $\mathrm{C} 11-\mathrm{C} 12-\mathrm{C} 13-\mathrm{C} 14$ & $-1.5(2)$ \\
\hline $\mathrm{C} 5-\mathrm{C} 6-\mathrm{C} 7-\mathrm{O} 2$ & $-1.18(19)$ & $\mathrm{C} 10-\mathrm{C} 9-\mathrm{C} 14-\mathrm{C} 13$ & $0.60(19)$ \\
\hline $\mathrm{C} 1-\mathrm{C} 6-\mathrm{C} 7-\mathrm{O} 2$ & $179.44(13)$ & $\mathrm{C} 8-\mathrm{C} 9-\mathrm{C} 14-\mathrm{C} 13$ & $-178.99(12)$ \\
\hline $\mathrm{C} 5-\mathrm{C} 6-\mathrm{C} 7-\mathrm{N} 1$ & $179.42(12)$ & $\mathrm{C} 12-\mathrm{C} 13-\mathrm{C} 14-\mathrm{C} 9$ & $0.7(2)$ \\
\hline $\mathrm{C} 1-\mathrm{C} 6-\mathrm{C} 7-\mathrm{N} 1$ & $0.04(18)$ & & \\
\hline
\end{tabular}

Hydrogen-bond geometry $\left(\AA,{ }^{\circ}\right)$

\begin{tabular}{lllll}
\hline$D-\mathrm{H} \cdots A$ & $D-\mathrm{H}$ & $\mathrm{H} \cdots A$ & $D \cdots A$ & $D-\mathrm{H} \cdots A$ \\
\hline $\mathrm{O} 1-\mathrm{H} 1 O 1 \cdots \mathrm{O} 2^{\mathrm{i}}$ & $0.80(2)$ & $1.92(2)$ & $2.7203(15)$ & $171(2)$ \\
$\mathrm{O} 3-\mathrm{H} 1 O 3 \cdots 2^{2 i}$ & $0.88(2)$ & $2.17(2)$ & $3.0276(13)$ & $163(2)$ \\
$\mathrm{O} 4-\mathrm{H} 1 O 4 \cdots \mathrm{O}^{\mathrm{iii}}$ & $0.82(2)$ & $1.93(2)$ & $2.7379(16)$ & $166(2)$ \\
$\mathrm{N} 1-\mathrm{H} 1 N 1 \cdots 3^{\mathrm{ii}}$ & $0.87(2)$ & $2.24(2)$ & $3.0017(16)$ & $146.1(19)$ \\
\hline
\end{tabular}

Symmetry codes: (i) $x,-y+1 / 2, z+1 / 2$; (ii) $-x+1,-y+1,-z$; (iii) $x-1, y, z-1$. 